Mots. Les langages du politique

\title{
La pauvreté multidimensionnelle. La dynamique sémantique dans le discours de la Banque mondiale
}

\section{Francine Mestrum}

\section{(2) OpenEdition}

\section{Journals}

Édition électronique

URL : https://journals.openedition.org/mots/14243

DOI : $10.4000 /$ mots. 14243

ISSN : 1960-6001

Éditeur

ENS Éditions

\section{Édition imprimée}

Date de publication : 1 novembre 2008

Pagination : 25-37

ISBN : 978-2-84788-147-9

ISSN : 0243-6450

Référence électronique

Francine Mestrum, « La pauvreté multidimensionnelle. La dynamique sémantique dans le discours de la Banque mondiale », Mots. Les langages du politique [En ligne], $88 \mid 2008$, mis en ligne le 01 novembre 2010, consulté le 23 avril 2022. URL : http://journals.openedition.org/mots/14243 ; DOI : https:// doi.org/10.4000/mots.14243 


\section{La pauvreté multidimensionnelle. La dynamique sémantique dans le discours de la Banque mondiale}

La Banque mondiale se présente comme une banque de savoir. Elle publie des rapports, des recherches, des statistiques et des recommandations et offre son expertise aux pays pauvres. En 1990, elle proposa de réorienter les politiques de développement vers la lutte contre la pauvreté. Entre 1990 et 1995, la Banque mondiale a publié dès lors un nombre important de documents dans lesquels elle a mis au point une conceptualisation de la pauvreté et des pauvres, ainsi qu'une stratégie de réduction de la pauvreté. Une analyse de ce discours révèle la parfaite correspondance entre ses représentations de la pauvreté et des pauvres, d'une part, et les politiques néolibérales qu'elle mène, mieux connues sous le nom de "Consensus de Washington», d'autre part (Mestrum, 2002).

Dans cet article, je présenterai d'abord la représentation multidimensionnelle de la pauvreté comme un élément clé du discours de la Banque mondiale. J'analyserai ensuite la façon dont celle-ci introduit de nouveaux concepts et comment elle joue sur la dynamique du sens de concepts existants. J'étudierai ensuite les mécanismes utilisés pour présenter son savoir. Enfin, je m'interrogerai sur la fidélité ou non de la Banque à un discours séculaire sur la pauvreté.

L'analyse du discours quej'ai mise en œuvre suit une approche sociologique qui appréhende le discours comme construction de savoir et comme acte communicationnel. Il s'agit d'une démarche interprétative qui a pour but d'expliquer la construction d'un savoir. Le sens du discours est donné par le locuteur et par le contexte, mais il peut être transformé dans les rapports interdiscursifs. La présente analyse ne vise pas à dévoiler ou à dénoncer une vérité cachée dans le discours, ni à confronter le discours à une quelconque vérité extradiscursive. Elle n'est qu'analyse d'un savoir et d'un acte communicationnel.

Le discours étudié a certes une fonction performative qui contribue à faire naitre ce qu’il énonce. En tant qu'acte communicationnel, il prétend à la vérité mais doit accepter d'être contredit. C'est en fonction de l'accueil réservé par l'allocutaire du discours que des stratégies divergentes, face au refus ou à

Université de Gand, Université libre de Bruxelles (GRAID), Francine.Mestrum@ulb.ac.be 
l'acceptation de la vérité proposée, peuvent être développées. Cette démarche appréhende le changement social à travers le discours et explique l'évolution des sociétés - entre autres - par l'évolution des savoirs des sociétés et des idées sur ce qui serait vrai.

\section{La multidimensionnalité de la pauvreté}

La multidimensionnalité de la pauvreté constitue l'une des cinq régularités qu'il est possible de repérer dans le discours des organisations internationales sur la pauvreté1. Les quatre autres étant la dimension temporelle qui associe la lutte contre la pauvreté au début d'une nouvelle ère de la coopération internationale, l'identification des pauvres comme étant les victimes des mauvais choix politiques du passé, la présentation des organisations internationales comme les acteurs principaux de la lutte contre la pauvreté, et l'insistance sur les opportunités créées par la libéralisation des échanges et par la mondialisation économique.

Selon la Banque mondiale, la conception de la pauvreté a évolué dans le temps et elle varie énormément d'une culture à l'autre. Elle est le reflet de priorités et de conceptions normatives du bien-être social et du droit propres à chaque pays ${ }^{2}$. La pauvreté est liée à un éventail très large de facteurs comprenant le revenu, la santé, l'éducation, l'accès aux biens, la position géographique, le genre, l'origine ethnique et les circonstances familiales. Cette nature multidimensionnelle de la pauvreté est difficile à mesurer3. Elle permet de prendre en compte un nombre illimité de problèmes, de causes autant que de conséquences et de symptômes de la pauvreté.

Cette multidimensionnalité permet aussi de situer les pauvres dans une altérité par rapport au reste de la société. En effet, la ligne de partage qui sépare les riches des pauvres ne concerne plus seulement les revenus, mais également des éléments aussi divers que la santé, l'éducation, le logement, la participation politique, etc.

Aussi pertinente que puisse être une telle approche, elle présente plusieurs écueils. D’abord, le problème de l'évaluation quantitative se complique singulièrement dès lors qu'on multiplie les indicateurs. Ensuite, cette approche risque de confondre les différentes dimensions avec la pauvreté elle-même et de reléguer à l'arrière-plan la question du revenu. Il devient ainsi parfaitement possible d'atteindre des taux satisfaisants dans tous les domaines non moné-

1. Le corpus étudié est constitué de l'ensemble des rapports de la Banque mondiale, de l'Organisation des Nations Unies (ONU) et du Programme des Nations Unies pour le Développement (PNUD) sur le thème de la pauvreté entre 1990 et 2006. Nous avons choisi de respecter la langue dans laquelle ces rapports ont été publiés.

2. Banque mondiale, Rapport sur le développement dans le monde 1990, Washington, p. 31.

3. The World Bank, 1996, Poverty Reduction and the World Bank, Washington, p. 2. 
taires de la pauvreté, sans éliminer pour autant la pauvreté monétaire et sans réduire l'écart de revenus entre les riches et les pauvres.

Dans son deuxième grand rapport sur la pauvreté4, la Banque définit celleci comme étant une vulnérabilité, un manque de voix et un manque d'empowerment. Ceci complique davantage la quantification.

Entre 1990 et 2000, on constate dans le discours de la Banque mondiale un glissement progressif vers les dimensions non monétaires de la pauvreté. Actuellement, le revenu est utilisé dans les statistiques, mais il n'y a pas de lien entre ces statistiques et son discours. Par ailleurs, ce lien n'a jamais réellement existé, dans la mesure où les statistiques mondiales sur la pauvreté étaient inexistantes au moment où la Banque, en 1990, commença à produire son savoir sur la pauvreté.

Cette non-prise en compte du revenu dans le discours et dans les stratégies de lutte contre la pauvreté contribue à en donner la responsabilité ultime aux pauvres eux-mêmes. Si les organisations internationales sont responsables de l'organisation de la lutte contre la pauvreté, celle-ci ne concerne que le contexte dans lequel les pauvres peuvent échapper à la misère.

\section{La dynamique sémantique}

La Banque mondiale introduit toute une série de mots nouveaux, souvent issus du vocabulaire managérial: stakeholder, governance, ownership, empowerment... L'avantage de ce procédé tient à ce que n'ayant pas de sens précis hors du contexte du management, ces mots peuvent facilement être intégrés à un discours technique qui ne viserait que l'efficacité. Cette neutralité politique favorise le consensus et ignore les conflits sous-jacents en matière de développement. De plus, elle limite le pouvoir des États de légiférer et d'imposer des mesures contraignantes. Ainsi, la gouvernance ne sera adéquate que dans la mesure où elle prendra en compte les partenaires sociaux (plus particulièrement les entreprises) et les pauvres, et où elle débouchera sur un accord volontaire. Les documents de la Banque mondiale ne parlent que très rarement des syndicats et presque jamais dans un sens positif5. La négociation entre les travailleurs du secteur formel et leur entreprise sur les conditions de travail et les avantages sociaux fait partie de la «bonne gouvernance», mais les droits économiques et sociaux des travailleurs n'en relèvent pas ${ }^{6}$.

L'ownership veut donner aux pays pauvres la responsabilité des politiques

4. The World Bank, World Development Report 2000/2001, Washington.

5. The World Bank, World Development Report 2006, Washington, p. 189.

6. The World Bank, Doing Business 2006: cette publication donne un classement des pays en fonction, entre autres, de l'inexistence d'un marché du travail régulé (jours fériés, durée du travail, licenciements...). 
à mettre en œuvre. Cependant, les grands axes macroéconomiques sont placés hors débat et les choix des États ne s'exercent qu'à leur superficie :

We have learned that a wide variety of approaches can work, but we've also learned that the key ingredients to successful economic growth include giving greater space to the private sector, promoting the rule of law and a functioning judiciary. [...] The Bank is willing to support a country's agenda, if we think the policies are sound, workable and truly owned by the government and its citizens7.

La Banque est disposée à céder la responsabilité aux pays pauvres, mais elle sait ce qu'ils ont à faire et elle continue de poser des conditions à ses financements ${ }^{8}$.

Bonne gouvernance et ownership sont des concepts particulièrement efficaces. Ils créent l'impression d'être neutres et de laisser aux pays pauvres la parfaite maitrise de leur politique de développement. Mais en réalité, ce sont les institutions de Bretton Woods qui continuent de définir les grandes lignes politiques et d'en contrôler la mise en œuvre.

Cette mainmise politique est facilitée par le nouveau sens que donne la Banque à des concepts existants.

\section{Le développement}

Selon les théories élaborées essentiellement à l'ONU dans les années soixante et soixante-dix, le développement était avant tout économique et toutes les revendications des pays pauvres se centraient sur la nécessité de l'indépendance économique pour renforcer l'indépendance politique. Bien que le développement social et politique ne fût pas absent du discours de l'ONU, il n'a jamais reçu la même attention que l'économie. La pensée sur le développement est clairement énoncée dans les grandes résolutions de l'assemblée générale de l'ONU, qu'il s'agisse des «décennies» pour le développement, de celles sur le nouvel ordre économique international, sur les droits et les devoirs économiques des États, sur la coopération économique, etc. 9 Certes, plusieurs théories divergentes étaient discutées, mais les grandes revendications économiques des pays pauvres concernaient l'industrialisation, le commerce équitable (meilleurs termes de l'échange), des prix justes pour les matières premières, le contrôle sur les multinationales, le transfert technologique, une part plus importante dans le secteur des services et une augmentation de l'aide au développement.

7. The World Bank, août 2004, «Development policy lending replaces adjustment lending», www.worldbank.org

8. Eurodad, «World Bank and IMF conditionality: a development injustice», working paper, juin 2006.

9. Voir résolutions des assemblées générales de l'ONU 1710 (XVI), 2626 (XXV), 3201 (S-VI), 3202 (S-VI), 3281 (XXIX), 3362 (S-VII), 35/56. 
À l'exception de ce dernier point, toutes ces revendications ont disparu du discours contemporain des organisations internationales. Actuellement, le développement se réfère, d'une part, à la croissance du marché mondial, devenu le référent central de la Banque mondiale et, d'autre part, à la lutte contre la pauvreté au niveau individuel. Le développement ne concerne plus les États, ni les nations, ni les peuples. Tant l'économie que le niveau national ont disparu du discours. L'économie fait désormais partie de la nature, elle n'est plus un domaine d'intervention: «Le développement obéit à des forces dont on est loin de comprendre pleinement le jeu. $»^{10} \|$ suffit de bien l'observer et de respecter ces forces. La dernière définition du développement économique formulée par la Banque mondiale date de 1991 et elle est plus sociale qu'économique: "[...] un relèvement durable du niveau de vie, celuici étant mesuré non seulement par le niveau de la consommation, mais aussi par le niveau d'instruction et l'état sanitaire de la population, ainsi que par le degré de protection de l'environnement ${ }^{11}$. Dans son Agenda pour le développement, l'ONU donne également une définition non économique du développement ${ }^{12}$. Aujourd'hui, développement, lutte contre la pauvreté et croissance sont utilisés comme s'il s'agissait de synonymes.

Les États ont également cessé d'être les acteurs du développement. Dans les pays pauvres, ils ont pour tâche l'insertion dans le marché mondial et l'aide aux pauvres. Ils doivent pour cela créer un environnement apte à attirer les investissements étrangers et promulguer des législations qui permettent au marché de faire son travail: lois sur la concurrence, sur la protection de la propriété, etc. Les États sont devenus des acteurs économiques qui ne disposent que d'une marge de manœuvre limitée: «L'expérience [...] a montré que l'État ne peut pas tenir ses promesses, son rôle doit être redéfini et orienté vers l'efficacité »13; «Il vaut mieux ne pas demander à l'État de gérer le développement. . ${ }^{14}$

Ce changement d'interprétation se retrouve dans les statistiques de la Banque mondiale. Dans ses rapports annuels sur le développement dans le monde, les pays ne sont plus classés en fonction de leur revenu, mais dans un ordre alphabétique sous la rubrique «Taille de l'économie». L'économie est appréhendée dans sa dimension mondiale. Ce n'est plus l'écart de revenu qui distingue les pays les uns des autres, mais leur degré d'intégration à l'économie mondiale et la façon dont ils organisent l'espace de la concurrence. Aujourd'hui, la voie du développement, tel qu'il était compris dans les années soixante et soixante-dix, se sépare en deux branches: d'une part, le marché

10. Banque mondiale, Rapport surle développement dans le monde, 1991, Washington, p. 1.

11. Ibidem, p. 38.

12. ONU, 1997, Rapport du groupe de travail ad hoc de l'Assemblée générale, à composition non limitée, chargée d'élaborer un Agenda pour le développement (Doc. A/AC.250/1), Rés. A.G. A51/45 du 19 septembre.

13. Banque mondiale, Rapport sur le développement dans le monde, 1997, Washington, p. iii.

14. Banque mondiale, 1991, op. cit., p. 4 . 
mondial, de l'autre, la lutte contre la pauvreté. Du développement des pays sous-développés, on est passé au développement des échanges commerciaux et au développement du «capital humain » des individus.

\section{La protection sociale}

La notion de protection sociale se transforme elle aussi. Sous sa forme actuelle, à la Banque mondiale, elle n'a plus rien à voir avec les concepts d'État providence, de citoyenneté sociale ou même tout simplement de politique sociale.

Le processus a commencé par la délégitimation de l'idée de sécurité sociale. Selon la Banque mondiale, les mécanismes traditionnels de protection des pauvres se sont révélés inefficaces: «[...] des solutions inédites associant les entreprises, les travailleurs, les ménages et les groupes de proximité doivent être trouvées pour assurer une plus grande sécurité à moindre coût ${ }^{15}$. Les salaires minimaux, par exemple, n'aident que ceux qui ont du travail mais laissent de côté ceux qui en cherchent. En général, selon la Banque, les mesures qui entravent le fonctionnement du marché ne protègent pas les pauvres ${ }^{16}$. L'accès égalitaire aux prestations de la sécurité sociale ne répond pas aux besoins d'une économie de marché17. Le PNUD partage cette vision. Il prétend que la sécurité sociale ne représente pas le meilleur emploi qu'un pays en développement puisse faire des ressources dont il dispose ${ }^{18}$. "La réduction de la pauvreté tend encore à être identifiée avec la sécurité sociale ou la protection sociale [...] partant peut-être de bons sentiments mais inefficaces ${ }^{19}$. Selon les organisations internationales, les dépenses sociales ne doivent s'adresser qu'aux pauvres, tandis que les privilégiés peuvent s'acheter une assurance sur le marché privé.

Une fois ces idées intégrées au consensus mondial créé autour de la lutte contre la pauvreté, la Banque a commencé à formuler des propositions pour une nouvelle protection sociale ${ }^{20}$. Celle-ci est placée dans le contexte d'une gestion de risques. Les pauvres et les riches étant égaux devant des risques identiques pour les deux groupes, force est de constater que les pauvres apparaissent nettement plus vulnérables. Ils n'ont en effet qu'un

15. Banque mondiale, 1997, op. cit., p. 6.

16. The World Bank, 1993, Poverty Reduction Handbook, Washington, p. 3, 31, 34, 38.

17. Banque mondiale, 1996, Rapport sur le développement dans le monde. De l'économie planifiée à l'économie de marché, Washington, p. 94.

18. PNUD, 1991, Rapport mondial sur le développement humain, Paris, Economica, p. 55.

19. PNUD, 2000, Vaincre la pauvreté humaine, New York, p. 42-44.

20. Holzmann, Jørgensen, 2000, Gestion du risque social : cadre théorique de la protection sociale, Document de travail $n^{\circ} 006$ sur la protection sociale, Washington, Banque mondiale, février; World Bank, 2000, Social Protection Sector Strategy. From Safety Net to Springboard, Washington, The World Bank. 
accès limité aux instruments de gestion des risques. C'est ce qui explique, selon la Banque, leur réticence à entreprendre des activités à haut risque et à haut rendement. Par conséquent, une nouvelle politique s'impose. Les gens pauvres bénéficient généralement de mécanismes de solidarité, mais ceux-ci sont trop peu efficaces et trop chers. C'est pourquoi la Banque veut les améliorer, car si la nature et l'économie sont immuables, nous ne sommes pas pour autant condamnés à l'impuissance. Nous pouvons et devons apprendre à nous protéger efficacement: «La protection sociale est l'ensemble des politiques publiques visant à i) aider les individus, les ménages et les collectivités à mieux gérer le risque, et ii) fournir un appui aux personnes extrêmement pauvres ${ }^{21}$. Elle est un filet de sécurité et en même temps elle fonctionne comme un tremplin. Elle est investissement, plus que facteur de coût.

La lutte contre la pauvreté n'est qu'un élément de la protection sociale qui, à son tour, n'est qu'un élément de la gestion du risque. La prévention des risques commence par une bonne politique macroéconomique, bien que des chocs restent possibles. Il est financièrement non soutenable de prévenir tous les risques, dit la Banque. Il est préférable de les atténuer, moyennant des investissements dans le capital humain et social, par des systèmes de microfinancement et des mécanismes de solidarité communautaire. Si un risque se concrétise, une aide directe mais temporaire aux personnes extrêmement pauvres est possible.

Le seul objectif de cette protection sociale est d'améliorer la capacité de gestion des individus. Les prestations monétaires et les garanties de revenu sont exclues. La redistribution des revenus peut être un résultat de la gestion des risques, mais elle n'en est pas l'objectif. Voilà l'importance d'une définition de la pauvreté qui ignore les revenus. Le revenu, disent Holzmann et Jørgensen, est un "phénomène stochastique »22. La lutte contre la pauvreté n'est pas une affaire de politiques sociales mais relève des politiques macroéconomiques, de la libéralisation des échanges et des équilibres budgétaires.

Ainsi, la Banque mondiale est en mesure de proposer, à la place de la sécurité sociale traditionnelle, une protection sociale au coût allégé et au rendement élevé en croissance économique. Cette gestion des risques ne vise aucun changement social, mais seulement la cohésion sociale. Ce qui doit être protégé, ce n'est pas le revenu mais la capacité des gens à créer des revenus. Le revenu devient une responsabilité des individus eux-mêmes. Celui qui prend des risques gagnera plus. La redistribution organisée ne peut être qu'arbitraire: «L'assurance n'est plus qu'un moyen parmi d'autres, et souvent pas le meilleur, de se protéger, et il n'est d'ailleurs pas possible de s'assurer contre

21. Holzmann, Jørgensen, op. cit., p. 11.

22. Ibidem, p. 7. 
de nombreux risques. ${ }^{23}$ Les inégalités sont la conséquence des efforts inégaux des individus et des forces - impersonnelles - du marché.

\section{Le développement social}

Le troisième exemple concerne le développement social à propos duquel la Banque a publié des documents en 2000 et en $2004^{24}$. Elle définit le développement social comme un moyen de réduire la pauvreté et de favoriser le développement. Le développement est mis en rapport avec la prévention des conflits (de la violence conjugale aux guerres civiles en passant par la criminalité ordinaire...) et avec le développement durable. Une réduction durable de la pauvreté, dit la Banque, est indissociablement liée à un changement sociétal positif, moyennant la promotion de normes et de valeurs, de règles formelles et informelles qui rendent les structures sociales «inclusives» et favorisent la cohésion sociale. Cette approche plutôt culturaliste ignore totalement les rapports de pouvoir et l'organisation économique des sociétés. La «durabilité sociale » devient un «actif» dans le portefeuille qu'ont à gérer les États. Si le capital social est menacé, les États doivent intervenir pour éviter des conflits, comme ils doivent le faire pour garantir la durabilité écologique (Mestrum, 2003).

Or, dans le passé, le développement social était vu comme le moyen de dépasser la dualité sociale, grâce aux interventions publiques dans le domaine social et économique. Le but ultime était le bien-être général et la lutte contre les inégalités. Certes, par rapport aux débuts de l'application du «Consensus de Washington» dans les années quatre-vingt, l'on assiste à la Banque mondiale à un certain retour de l'État, mais quel État? La Banque a toujours attaché une certaine importance au développement institutionnel, moins d'un point de vue politique que par souci d'efficacité des politiques de développement. Les politiques d'ajustement structurel ont systématiquement affaibli les États dans leur rôle économique et social. À partir de 1990, est attribuée à l'État la responsabilité de défendre l'intérêt commun, notamment par la lutte contre la pauvreté. Mais pour cela, l'État et le marché doivent se tendre la main, selon le PNUD25. Toutefois, l'État est constamment revêtu de caractérisations à connotation négative telles qu'inertie, bureaucratie, arbitraire, manque d'efficacité.

Aujourd'hui, la Banque prône la «bonne gouvernance», car la pauvreté persistante dans les pays en développement est de plus en plus attribuée

23. Holzmann, Jørgensen, op. cit., p. 12.

24. The World Bank, 2000, New Paths to Social Development. Community and Global Networks in Action, Washington, The World Bank; idem, 2004, Social Development in World Bank Operations : Results and Way Forward.

25. PNUD, 1993, Rapport mondial surle développement humain, Paris, Economica, p. 55. 
précisément à une gouvernance défaillante. Or, cette approche subit de plus en plus une «culturalisation». Le Global Competitivity Report 2004-2005 du Forum économique mondial utilise les mêmes exemples que Huntington dans ses études sur le développement: "Culture is the mother, institutions are the children.» (Huntington, Harrison, 2000, xxviii)

Avec les politiques d'ajustement structurel, l'autonomie économique des pouvoirs publics a été amoindrie fortement en mettant ainsi fin aux rêves du développement. Avec la lutte contre la pauvreté, la sécurité sociale devrait être éliminée. Avec la protection sociale, on met en place une «gestion de risque » et on encourage, voire oblige, tous les individus à se lancer sur un marché du travail dérégulé. Avec le développement social et la bonne gouvernance, on culturalise ainsi la pauvreté.

\section{Les mécanismes discursifs}

La Banque mondiale n'avançait aucune justification concrète à son introduction de stratégies de lutte contre la pauvreté en 1990; elle parlait d'«impératif moral» et définissait la pauvreté essentiellement comme un déficit de développement et toute une série de phénomènes qui la caractériseraient. Son raisonnement était plutôt paradoxal, dans la mesure où elle condamnait les politiques de développement du passé, tout en vantant les succès du développement des trois décennies antérieures, en termes économiques et d'indicateurs sociaux ${ }^{26}$. De plus, à l'époque, son approche de la pauvreté ne reposait sur aucun fondement empirique. Ses calculs d'un seuil de pauvreté de 1 \$ par jour ont fait l'objet de critiques importantes (Reddy, Pogge, 2002; Went, 2004) et, depuis, elle a plusieurs fois changé de méthode. En 1980, elle estimait le nombre de pauvres extrêmes dans le monde en développement à 800 millions de personnes. Aujourd'hui, elle réévalue ses chiffres concernant le début des années quatre-vingt et parle, pour cette époque-là, de 1,481 milliard de pauvres extrêmes. Grâce au relèvement de ce chiffre, elle peut s'enorgueillir d'une réduction de moitié de la proportion de pauvres entre 1981 et 200127. Cet exemple ne sert qu'à démontrer les variations méthodologiques de la Banque. La pauvreté étant très difficile à mesurer, ces chiffres ne sont sans doute pas plus corrects ou incorrects que d'autres. Cependant, chaque définition, chaque fixation d'un seuil de pauvreté traduit évidemment les valeurs de son auteur. La Banque est la seule organisation qui s'efforce de calculer et de publier des statistiques pour l'ensemble du monde en développement et nous n'avons que ses chiffres pour parler de la pauvreté mondiale.

26. Un exemple: Banque mondiale, 1990, op. cit., p. 1; idem, 1997, op. cit., p. iii, 1-2.

27. Chen, Ravallion, 2004, "How have the world's poor fared since the early 1980s? ", World Bank Policy Research Paper 3341, juin. 
Les caractéristiques néolibérales des politiques proposées par la Banque au nom de la lutte contre la pauvreté laissent moins de doutes. Certes, la lutte contre la pauvreté étant un thème consensuel par excellence, il n'est dès lors pas trop difficile d'imposer en son nom des politiques d'ajustement structurel. Pour autant, les résultats positifs ne peuvent éternellement se faire attendre. La restructuration économique dont s'accompagne l'ajustement est parfaitement conforme à la stratégie de lutte contre la pauvreté. À court terme toutefois, beaucoup de pauvres risquent d'en souffrir, selon la Banque ${ }^{28}$. C'est ce qui explique les mécanismes discursifs utilisés, la Banque ne pouvant chercher les causes du retard dans le système économique lui-même. Dès lors, elle a recours aux formulations probabilistes et à un schéma constamment répété : abandonner les savoirs du passé, dont nous savons aujourd'hui qu'ils ne sont pas bons, et tenir compte des leçons de l'histoire. L'avenir sera sans aucun doute meilleur qu'aujourd'hui à condition d'introduire des politiques "saines». Mais la Banque reste extrêmement prudente et truffe son discours d'expressions qui permettent toutes les issues (en général, en moyenne, très souvent) tout en se référant à des «recherches» qui démontreraient le bienfondé de ce qu'elle veut imposer. Voici quelques exemples extraits du Rapport sur le développement dans le monde $2002^{29}$ et d'un rapport sur l'aide au développement30:

[...] the importance of the historic context: where countries are today affects where they can go (WBo2, p. iii)

History shows that politics influences the development of financial systems. (WBo2, p. 22)

History reveals the political necessity for more sophisticated laws. (WBo2, p. 64)

Effective institutions can make a difference in the success of market reforms. (WBo2, p. iii)

Many studies have documented strong associations between per capita income and measures of the strength of property rights. (WBo2, p. 99)

There is evidence that excessive regulation undermines economic growth. (WBo2, p. 99)

There is growing evidence that countries that are more open to international trade have lower corruption. (WBo2, p. 107)

A $1 \%$ increase in GDP/capita tends in average to lead to a $1 \%$ increase in the incomes of the poorest quintile of the population. (ASS, p. 40)

28. Banque mondiale, 1990, op. cit., p. 3, p. 119.

29. The World Bank, 2002, World Development Report. Building Institutions for Markets, Washington (WBo2).

30. The World Bank, 2002, The Role and Effectiveness of Development Assistance, Washington (ASS). 
In the right environment, with the right design, adjustment lending has been broadly effective in spurring development. (ASS, p. 39)

Policies such as trade openness, low inflation, moderate size of government and strong rule of law have generally benefited the income of poor people. (ASS, p. 40)

World Bank assistance is, on average, well targeted at poverty reduction. (ASS, p. 45)

The Bank's actions have been broadly successful. (ASS, p. 60).

En conclusion, la Banque ne cesse d'affirmer que «[the] development is a risky business and some failures are inevitable» (ASS, p. 57), ou encore : "We have learned that development is possible, not inevitable "31, et "The only thing that can be said with certainty about the future, is that itwill differfrom the present $॥ 32$. Voilà des expressions qui lui permettent, à tout moment, d'échapper à une évaluation critique de ses politiques et d'attribuer la responsabilité de l'échec des politiques aux pays pauvres qui n'ont pas de bonne gouvernance.

\section{Des léproseries à la pauvreté multidimensionnelle}

Au Moyen Âge, à partir de la fin $d u 12^{\mathrm{e}}$ siècle, presque chaque ville européenne avait sa léproserie. Y étaient enfermés les malades qui étaient de cette façon exclus de la société. À la même époque commençaient à être créés les hôpitaux où étaient accueillis des pauvres, des pèlerins et des malades (Goglin, 1976, p. 159). Vers la fin du Moyen Âge, au $14^{\mathrm{e}}$ siècle, les léproseries vont disparaitre, mais les valeurs et les images qui s'étaient attachées au personnage du lépreux sont restées (Foucault, 1972, p. 13).

Cela témoigne d'une constante dans l'histoire de la pauvreté et des pauvres en Europe. La pauvreté n'est jamais pensée seule, elle est toujours associée à d'autres «vices» qui méritent l'exclusion. Pourtant, en même temps, les pauvres sont spirituellement intégrés à la société. C'est au $15^{\mathrm{e}}$ siècle qu'apparaissent les "nefs des fous », compositions artistiques et littéraires mais aussi réalité objective. Dans l'œuvre de Sébastien Brant, la nef des fous transporte «des avares, des délateurs, des ivrognes, ceux qui se livrent au désordre et à la débauche, ceux qui interprètent mal l'Écriture, ceux qui pratiquent l'adultère ». Bref, c'est l'exclusion de la société de toutes les irrégularités de conduite (Foucault, 1972, p. 36).

Dès la fin $\mathrm{du} 16^{\mathrm{e}}$ siècle et surtout au $17^{\mathrm{e}}$ siècle, toute cette compagnie sera enfermée dans les hôpitaux et les anciennes léproseries, qui deviennent vite

31. James D Wolfensohn, 1999, "Coalitions for change. Address to the board of governors » [of the World Bank], Washington D.C., 28 septembre.

32. The World Bank, World Development Report 1999/2000. Entering the 21st Century, Washington, p. 28. 
des lieux de travail forcé. Selon Foucault, cet «amalgame abusif d'éléments hétérogènes» s'explique par l'éclosion d'une nouvelle sensibilité à la misère et aux devoirs de l'assistance, une nouvelle forme de réaction devant les problèmes économiques du chômage et l'oisiveté, une nouvelle éthique du travail (Foucault, 1972, p. 66). La mendicité et l'oisiveté étaient considérées comme les sources de tous les désordres et le monde ouvrier était désorganisé par l'apparition de nouvelles structures économiques. L'internement et la nouvelle approche de la pauvreté - et d'autres formes d'irrégularités - s'expliquent donc par les besoins des non-pauvres.

Ce seul exemple - parmi beaucoup d'autres (Sassier, 1990; Geremek, 1987) - aide à comprendre que la Banque mondiale n'a rien inventé dans ses stratégies de réduction de la pauvreté et que son discours s'inscrit parfaitement dans une pensée séculaire qui fait de la pauvreté une construction sociale au service des non-pauvres, voire des riches (Simmel, 1998). Aujourd'hui, la pauvreté n'est pas non plus associée à un manque de ressources, mais à une «mauvaise gouvernance» qui empêche le pauvre d'entrer sur le marché du travail et qui pourrait nécessiter, un jour, un redressement par des interventions «humanitaires».

Les réformes proposées par la Banque mondiale, au nom d'une réduction de la pauvreté, sont toutes orientées vers la mise en place d'une mondialisation politique dans laquelle les institutions de Bretton Woods veillent au respect des dogmes néolibéraux. De plus, elles visent la mise en place de sociétés duales en éliminant les classes moyennes et les élites nationalistes des sociétés et en faisant des pauvres les alliés des riches mondialisés. Dans le nouvel ordre mondial désiré par la Banque mondiale, les pauvres sont des acteurs sociaux qui œuvrent pour le changement et qui participent à une gouvernance consensuelle. Comme au Moyen Âge, nous nous dirigeons vers une société d'humiles et de potentes (Goglin, 1976, p. 9).

Parler de la pauvreté et du rôle des pauvres dans le changement social est une métaphore pour parler de l'ordre social où les riches et les pauvres ont leur place. Les pauvres eux-mêmes sont enrôlés dans la défense de cet ordre social où l'on fait comme si la richesse elle-même, comme la pauvreté, n'avait rien à voir avec le niveau de revenu. La lutte contre la pauvreté n'introduit pas seulement un nouveau paradigme social - en éliminant la perspective de l'État providence et de la citoyenneté sociale -, elle réoriente également le discours politique vers une légitimation des politiques macroéconomiques, mises hors champ du discutable. On assiste à une naturalisation de l'économie et à la création d'une pauvreté non matérielle qui permet l'amalgame de toutes les catégories sociales jugées inutiles. Le pauvre devient soit l'homo œconomicus empêché par l'État, et ses politiques erronées, de jouer son rôle économique, soit l'asocial, l'inutile qui rejoint les classes dangereuses. La pau- 
vreté multidimensionnelle permet d'installer tous les asociaux à la périphérie de la société où soit ils seront une source de main-d'œuvre bon marché, soit, à défaut de pouvoir se valoriser, ils rejoindront la classe des inutiles au monde, virtuellement embarqués sur une nef des fous.

\section{Références}

Foucault Michel, 1972, Histoire de la folie à l'âge classique, Paris, Le Seuil.

GEREMEK Bronislaw, 1987, La potence ou la pitié, Paris, Gallimard.

Goglin Jean-Louis, 1976, Les misérables dans l'Occident médiéval, Paris, Le Seuil.

Huntington Samuel, Harrison Lawrence I. éd., 2000, Culture Matters. How Values

Shape Human Progress, Basic Books.

Mestrum Francine, 2002, Mondialisation et pauvreté. De l'utilité de la pauvreté dans le nouvel ordre mondial, Paris, L'Harmattan.

- 2003, "Poverty reduction and sustainable development», Environment, Development and Sustainability, $\mathrm{n}^{\circ}$ 5, p. 41-61.

Reddy Sanjay G., Pog Ge Thomas W., 2002, «How not to count the poor», www.socialanalysis.org

SASSIER Philippe, 1990, Du bon usage des pauvres, Paris, Fayard.

SIMMEL Georg, 1998, Les pauvres, Paris, PUF.

WENT Robert, 2004, «How not to measure global poverty and inequality», Amsterdam, INEM Conference. 